\title{
A Reappraisal of Sedimentation Nonideality Coefficients for the Analysis of Weak Interactions of Therapeutic Proteins
}

\author{
Sumit K. Chaturvedi ${ }^{1}$ and Peter Schuck ${ }^{1,2}$
}

Received 11 January 2019; accepted 11 February 2019; published online 27 February 2019

\begin{abstract}
The study of weak or colloidal interactions of therapeutic proteins in different formulations allows prediction and optimization of protein stability. Various biophysical techniques have been applied to determine the second osmotic virial coefficient $B_{2}$ as it reflects on the macromolecular distance distribution that governs solution behavior at high concentration. In the present work, we exploit a direct link predicted by hydrodynamic theory between $B_{2}$ and the nonideality of sedimentation, commonly measured in sedimentation velocity analytical ultracentrifugation through the nonideality coefficient of sedimentation, $k_{S}$. Using sedimentation equilibrium analytical ultracentrifugation for independent measurement of $B_{2}$, we have examined the dependence of $k_{S}$ on $B_{2}$ for model proteins in different buffers. The data exhibit the expected linear relationship and highlight the impact of protein shape on the magnitude of the nonideality coefficient $k_{S}$. Recently, measurements of $k_{S}$ have been considerably simplified allowing higher throughput and simultaneous polydispersity assessment at higher protein concentrations. Thus, sedimentation velocity may offer a useful approach to compare the impact of formulation conditions on weak interactions and simultaneously on higher-order structure of therapeutic proteins.
\end{abstract}

KEY WORDS: hydrodynamics; protein-protein interactions; protein-solvent interactions; sedimentation velocity; virial coefficients.

\section{INTRODUCTION}

Protein pharmaceuticals are a rapidly expanding class of therapeutics. In particular, monoclonal antibody products have found broad application with an increasing number of targets (1-3). One of the challenges is the development of formulations that provide colloidal stability, low solution viscosity, and ensure the absence of immunogenic aggregates at very high protein concentrations (4-7). Therefore, techniques that can assess both the formation of higher-order structure and measure weak particle interactions are of great interest in this field. In particular, it has been shown that the second virial coefficient $B_{2}$ can correlate with aggregation, viscosity, solubility, and liquid-liquid phase separation (8-15). Techniques used to measure $B_{2}$ include small angle scattering, static light scattering (SLS), sedimentation equilibrium (SE) and sedimentation velocity (SV) analytical ultracentrifugation (AUC), self-interaction chromatography, osmometry, and

\footnotetext{
${ }^{1}$ Dynamics of Macromolecular Assembly Section, Laboratory of Cellular Imaging and Macromolecular Biophysics, National Institute of Biomedical Imaging and Bioengineering, National Institutes of Health, 13 South Drive, Bldg. 13, Rm 3N17, Bethesda, Maryland 20892, USA.

${ }^{2}$ To whom correspondence should be addressed. (e-mail: Peter.Schuck@nih.gov)
}

dynamic light scattering (DLS) in combination with SV $(9,13,15-26)$.

Unfortunately, measurement of the second osmotic virial coefficient is challenging with any technique, and difficulties are exacerbated by sample polydispersity. For example, SLS is a very attractive method due to the ability to measure solutions at very high concentrations, even beyond the range where $B_{2}$ alone is sufficient to describe the concentrationdependent behavior and more precise analyses are necessary $(27,28)$. On the other hand, SLS signals are highly sensitive to trace aggregates and impurities of larger size, which may be encountered in concentrated solutions (10). Another light scattering approach is DLS, where the change in mutual diffusion coefficient $D$ with concentration $c$ can be measured through the nonideality coefficient of diffusion, $k_{D}$. As has been pointed out by Saluja and colleagues, through analysis of the autocorrelation function in DLS, it is possible to discriminate and exclude signals from large particles that would dominate SLS measurements (10). However, a caveat is that this does not work for oligomers and smaller aggregates that cannot be resolved in DLS, and that strictly the basic foundations of polydispersity analysis in DLS break down in the presence of hydrodynamic interactions (29). Conflicting results were reported from experiments probing the theoretically expected linear relationship between the nonideality coefficient of diffusion, $k_{D}$ and $B_{2}$. While Ghosh 
et al. observed poor correlation between $k_{D}$ by DLS and $B_{2}$ by SLS (27), two other studies report good correlations $(9,30)$. However, the relationship was not considered universal but molecular shape dependent (9).

The nonideality coefficient of diffusion and the second virial coefficient are intimately linked to the nonideality coefficient of sedimentation, $k_{S}$, as measured from the decreasing sedimentation velocity with higher concentrations, through the simple relationship $k_{D}=2 B_{2}-k_{S}(9,16,27,31,32)$ (considering $B_{2}$ in volume/weight units; analogous to $B_{2}{ }^{\prime} M$ with a second virial coefficient $B_{2}^{\prime}$ in molar units). Thus, the combination of DLS to measure $k_{D}$ and SV to measure $k_{S}$ has been used to determine $B_{2}(9,10,30)$.

Previous work in this field has not yet made use of the result from statistical fluid dynamics that hydrodynamic interactions that govern $k_{S}$ are directly dependent on the interparticle distance distribution, in a way that establishes a direct link between the reduction of sedimentation in nonideal solutions and the second virial coefficient without reference to diffusion nonideality (33-37). The question whether this can be exploited for weakly interacting proteins and to what extent shape-dependent (i.e., protein-dependent) parameters need to be considered in the interpretation of $k_{S}$ is examined in the present work. Examining weak interactions through nonideal SV seems attractive because, independently, SV is already widely used to quantitate the presence of immunogenic oligomers and higher-order structures in formulations of therapeutic proteins, orthogonal to chromatographic and other methods (29,38-41).

This problem gains more practical relevance since we have recently introduced a new method to determine nonideality coefficients of sedimentation simultaneous with high-resolution size distributions from single experimental data sets (29), which eliminates the need to run samples at multiple concentrations for determining $k_{S}$. The new method also increases the concentration limits for the quantitation of trace oligomers and aggregates by SV, providing information on higher-order structures closer to formulation conditions from the same experiment. Furthermore, we have recently shown that weak macromolecular self-association can be measured with this approach (42). In parallel, alternative detection modes (43) and the use of 3D printing technology (44) in the development of new centerpieces can enhance the throughput and reduce sample requirements of SV (manuscript submitted). Therefore, we believe the measurement of nonideality coefficients $k_{S}$ by SV can be an attractive approach for the efficient characterization of weak interactions and higher-order structure of therapeutic proteins in different formulation conditions.

\section{MATERIALS AND METHODS}

\section{Proteins}

NISTmAb (45) was purchased from NIST (SRM 8671) and studied in the original buffer of $25 \mathrm{mM}$ histidine, $\mathrm{pH} 6.0$, or was dialyzed into $25 \mathrm{mM}$ histidine buffer $\mathrm{pH} 6.0$ with $5 \mathrm{mM}$ or $50 \mathrm{mM} \mathrm{NaCl}$, respectively. VRC01 and VRC07-523LS were kindly provided by Drs. Jai Pathak, Lisa Kueltzo, and Frank Arnold. VRC01 was studied in $25 \mathrm{mM} \mathrm{Na-Citrate,}$ $50 \mathrm{mM} \mathrm{NaCl}, 150 \mathrm{mM}$ Arginine $\mathrm{HCl}, \mathrm{pH}$ 5.8, and VRC07-
523LS was studied in $50 \mathrm{mM}$ Histidine, $50 \mathrm{mM} \mathrm{NaCl}, 5 \% \mathrm{w} / \mathrm{v}$ sucrose, $2.5 \% \mathrm{w} / v$ sorbitol, $\mathrm{pH}$ 6.8. Hen egg lysozyme was purchased from Sigma (catalog \# L6876; Sigma Aldrich, St. Louis, MO) and dialyzed into $10 \mathrm{mM}$ acetate buffer $\mathrm{pH} 4.6$ with $10 \mathrm{mM}, 100 \mathrm{mM}$, or $300 \mathrm{mM} \mathrm{NaCl}$, respectively. Ovalbumin was purchased from Sigma (catalog \# A5503; Sigma Aldrich, St. Louis, MO) and dialyzed into $10 \mathrm{mM}$ sodium phosphate, $\mathrm{pH}$ 7.4, $150 \mathrm{mM} \mathrm{NaCl}$. Protein concentrations were measured refractometrically by Rayleigh interferometry in the analytical ultracentrifuge.

\section{Analytical Ultracentrifugation}

SE and SV experiments were carried out in an Optima XL-A/I (Beckman Coulter, Indianapolis IN) following standard techniques $(46,47)$ unless mentioned otherwise. For SE, cell assemblies were mechanically stabilized by repeated exposure to strong centrifugal fields. Buffer blanks for SE were measured at one or multiple equilibrium rotor speeds (9000 rpm and 14,000 rpm for antibodies; 25,000 rpm or $27,000 \mathrm{rpm}$ or $50,000 \mathrm{rpm}$ for lysozyme; $18,000 \mathrm{rpm}$ for ovalbumin). Samples were filled into cell assemblies with $3 \mathrm{~mm}$ or $12 \mathrm{~mm}$ pathlength Epon double sector centerpieces at volumes to achieve $\approx 4 \mathrm{~mm}$ or $\approx 12 \mathrm{~mm}$ solution column heights for SE or SV, respectively. The rotor was temperature equilibrated to a nominal temperature of $19.7^{\circ} \mathrm{C}$ while resting in the rotor chamber prior to the start of centrifugation. For SE, an overspeeding schedule was calculated to minimize the equilibration time (48); for SV the rotor was accelerated to full speed at once $(40,000 \mathrm{rpm}$ for VRC antibodies, 45,000 rpm for NISTmAb, 50,000 rpm for all other). Rayleigh interference optical data acquisition was used. All data analyses were carried out with models implemented in SEDFIT, SEDPHAT (available at sedfitsedphat.nibib.nih.gov), and MATLAB (Mathworks, Natick, MA). Statistical error analyses were based on F-statistics with a $P$ value of 0.95 (49) (SE analysis) or via standard $\chi^{2}$ error analysis of linear regression (50) (SV analysis).

\section{Sedimentation Velocity Analysis}

With experiments predating the recently introduced nonideal sedimentation coefficient distribution approach (29), all data were analyzed using the traditional two-step procedure: first, scan sets were fitted with the standard sedimentation coefficient distribution $c(s)(51,52)$. Integration of the monomer peak was carried out to determine the weight-average sedimentation coefficient $s_{w}$, and, from the data at the lowest protein concentration approximating ideal dilution, the best-fit frictional ratio $f / f_{0}$. This determination of $s_{w}$ is equivalent to considerations of mass transport through a plane in the plateau region and independent of radial position, normalized to transport conditions at the start of centrifugation $(52,53)$. However, since samples undergo slight radial dilution in the sector-shaped sample (54), the corresponding sample concentration was calculated as the weighted time-average plateau concentration previously introduced for self-associating systems $(32,53)$. An uncertainty arises from the mist-fit of the standard sedimentation coefficient distribution $c(s)$, and the impact on $s_{w}$ can be estimated by calculating mass transport implied by residuals 
of the fit. Because the residuals are largely symmetrical around the inflection point, this misfit typically amounted to errors in $s_{w}$ values $<10^{-3} \mathrm{~S}$.

In a second analysis step, nonideality coefficients $k_{S}$ were calculated from $s_{w}$ as a function of concentration by fitting

$s(w)=s(0)\left(1-k_{S} w\right)$

where $w$ denotes the protein weight concentration. (Alternatively, the lysozyme sedimentation scans were fitted with nonideal sedimentation coefficient distribution $c_{\mathrm{NI}}\left(s_{0}\right)$ to determine $k_{S}$ directly $(29,42)$.) For the purpose of evaluating $k_{S}$ from the relative reduction of the sedimentation velocity, it is not necessary to normalize sedimentation coefficient data to conditions in water at $20^{\circ} \mathrm{C}$ (neither is it necessary to apply small calibration factors $(55,56)$ for this purpose). In this regard, it should be noted that an alternate approach of applying solution density corrections leads to an analogous framework but with nonideality coefficients $k_{S}^{\prime}$ reduced by the partial-specific volume $\bar{v}$, as discussed by Harding and Johnson (31). The framework of uncorrected $s$ values used in the present work corresponds naturally to the nonideal sedimentation coefficient distributions $c_{N I}\left(s_{0}\right)$ (29). It also matches the statistical fluid mechanics treatment of sedimentation by Batchelor $(37,57)$, which for non-interacting hard spheres in the dilute regime predicts the linear relationship

$s(\Phi)=s(0)(1-6.55 \Phi)$

where $\Phi$ denotes the volume fraction occupied by the sedimenting particle (57). This expression has been generalized by Batchelor and Wen (37) for weakly interacting spheres in the dilute regime

$s(\Phi)=s(0)\left(1-3.03 \Phi-3.52 B^{*} \Phi\right)$

with the reduced second virial coefficient $B^{*}$ defined as the ratio of $B_{2}$ and the hard-sphere virial coefficient $B_{H S}$ (33-37). Extensions for higher-order approximations suitable for higher volume fractions using a Baxter sticky-sphere approach were presented by Swan and colleagues (36).

It is worth highlighting the fundamental difference between Eq. 3 and the previously widely used relationship between $k_{S}$ and $B_{2}$ via the nonideality coefficient of diffusion $k_{D}=2 B_{2}-k_{S}(9,16,27,31,32)$. The latter can be derived on the basis of the virial expansion of the osmotic susceptibility, and therefore rests on thermodynamic considerations where no detailed molecular model is invoked, and where coefficients $k_{S}$ and $k_{D}$ may be considered phenomenological parameters of concentration dependence. By contrast, Eq. 3 rests on statistical fluid mechanics and the explicit calculation of hydrodynamic forces between sedimenting spheres, based on the particle distance distribution. This distance distribution is related to the interparticle potential and therefore linked to the second virial coefficient. It accounts, for example, for the probability of transient dimer formation (close proximity of two particles) that would diminish overall hydrodynamic drag $(37,58)$. Therefore, Eq. 3 does not require a reference to $k_{D}$.

To relate the linear coefficients in Eqs. 1 and 3, it is necessary to consider the connection between protein concentration and the relevant volume fraction. This is far from trivial and a long-standing problem for non-globular macromolecules $(31,59)$. If only the macromolecular volume excluded from solution is considered, the volume fraction relates directly to the protein partial-specific volume, $\bar{v}$ and with $\Phi=w \bar{v}$ one arrives at a prediction for the nonideality coefficient of sedimentation

$k_{S, V} \sim\left(3.03+3.52\left[B_{2} / B_{H S}\right]\right) \bar{v}$

However, theoretical work for nonideality coefficients of non-spherical particles have revealed a particle shape dependence (31,60-62), and a large body of experimental work in SV has shown that nonideality coefficients of proteins strongly increase with macromolecular shape asymmetry $(62,63)$. In his theory of nonideal sedimentation, Rowe has proposed the relevant volume fraction to be that of the hydrodynamically equivalent sphere $(32,64)$, which leads to the relationship

$\Phi_{S}=w \bar{v}\left(f / f_{0}\right)^{3}$

with $f / f_{0}$ denoting the protein frictional ratio, such that $\Phi_{S}$ represents the total volume occupied by hydrodynamically equivalent spheres. We insert this consideration into the framework of Batchelor's relationships. In this approximation, the nonideality coefficient for sedimentation would be expected to follow

$k_{S, H} \sim\left(3.03+3.52\left[B_{2} / B_{H S}\right]\right) \bar{v}\left(f / f_{0}\right)^{3}$

as a function of both frictional ratio and second virial coefficient.

Whether relationship Eq. 4 or Eq. 6 provides a better description of experimental data for proteins is not clear. The attempt to use experimental $k_{S}$ values to decide between hydrodynamic volume models is complicated by the usually unknown contribution of particle interactions to $B_{2}$. Compounding these problems, it should be noted that for non-spherical particles $B_{H S}$ will also be a non-trivially shapedependent quantity (65). Still, we may unify both expressions Eqs. 4 and 6 into the form

$k_{S}=k_{S}^{0}\left(1+1.16\left[B_{2} / B_{H S}\right]\right)$

Under "theta conditions" where $B_{2}$ vanishes, the nonideality coefficient of sedimentation termed $k_{S}^{0}$, will be a reflection of the relevant hydrodynamic volume alone. In this way, the data sets of $k_{S}$ vs. $B_{2}$ should offer the opportunity to shed more light on this question. But in any event, the nonideality coefficient increases linearly with the second virial coefficient, which may be sufficient 
for comparative screening applications even without determining the absolute values of $B$.

\section{Sedimentation Equilibrium Analysis}

The radial concentration distributions of a single species in sedimentation equilibrium follow

$c(r)=c\left(r_{0}\right) \exp \left[\frac{M}{1+c(r)(\partial \ln \gamma / \partial c)(r)} \times \frac{(1-\bar{v} \rho) \omega^{2}}{R T}\left(r^{2}-r_{0}^{2}\right)\right]$

where $M$ is the molar mass, $\gamma$ the chemical activity coefficient, $R$ the gas constant, $T$ the temperature, $\rho$ the solvent density, $\omega$ the rotor angular velocity, and $r_{0}$ a reference radius $(21,66)$. At not too high concentrations, accounting only for the first term of the virial expansion of the activity coefficient leads to the implicit equation for the measured signal $a(r)$

$$
\begin{aligned}
a(r)= & c\left(r_{0}\right) \exp \left[\frac{M}{1+2 B_{2}^{\prime} M c(r)} \times \frac{(1-\bar{v} \rho) \omega^{2}}{R T}\left(r^{2}-r_{0}^{2}\right)\right] \\
& +b(r)
\end{aligned}
$$

which can be solved iteratively and may be superimposed by a baseline term $b(r)$. Alternatively, to account for a wider concentration range, the chemical activity as a function of occupied volume fraction may be approximated with the Carnahan-Starling formula

$\ln \gamma=\frac{8 \Phi-9 \Phi^{2}+4 \Phi^{3}}{(1-\Phi)^{3}}$

for suspensions of hard spheres $(67,68)$. Considering the protein as effective hard spheres of swollen radius with $\Phi=c M \bar{v}$ $\left(B_{2} / B_{H S}\right)$ and with the virial coefficient of non-interacting hard spheres $B_{H S}=4 \bar{v}$, we can extend Eq. 9 by inserting

$$
\frac{\partial \ln \gamma}{\partial c}=M \bar{v} \frac{B_{2}}{B_{H S}}\left[\frac{24 \Phi-27 \Phi^{2}+12 \Phi^{3}}{(1-\Phi)^{4}}+\frac{8-18 \Phi+12 \Phi^{2}}{(1-\Phi)^{3}}\right]
$$

in Eq. 8 (68). At low concentrations, it has the limiting value $8 M \bar{v}\left(B_{2} / B_{H S}\right)$, which is consistent with the value of $2 M B_{2}^{\prime}$ as in Eq. 9. At a volume fraction of $1 \%$, the difference to Eq. 9 amounts to $\approx 4 \%$ in $B_{2}$, increasing approximately proportionally to a volume fraction of $5 \%$. Equation 11 was implemented in SEDPHAT using the INVEQ approach (21). This model was fitted globally to multi-speed data sets at multiple loading concentrations, weighted with inverse loading concentration (69).

\section{RESULTS}

We chose the NISTmAb reference molecule in different buffers to examine the nonideality coefficient of sedimentation $k_{S}$ and its relationship to the second virial coefficient $B_{2}$.
For NISTmAb in $25 \mathrm{mM}$ histidine $\mathrm{pH} 6.0$ (in the formulation buffer that does not contain salt) from SE experiments, we obtained a best-fit estimate for $B_{2}$ of $27.1 \mathrm{ml} / \mathrm{g}(25.5-28.7 \mathrm{ml} /$ g; $95 \%$ confidence interval), which compares well with the value of $25 \mathrm{ml} / \mathrm{g}$ reported by others using SLS and SE in these conditions (70). Figure 1 shows typical multi-speed SE data for NISTmAb up to $13 \mathrm{mg} / \mathrm{ml}$ in $5 \mathrm{mM} \mathrm{NaCl}$, with the best-fit single nonideal species sedimentation model Eq. 8 based on effective hard spheres Eq. 11. SV data for the same set of conditions are shown in Fig. 2, with the best-fit linear regression to determine $k_{S}$ following Eq. 1. The estimated variance of the $s_{w}$ values was $0.03 \mathrm{~S}$, slightly above the expected experimental precision of $0.01 \mathrm{~S}$.

The nonideality coefficients are plotted against $B_{2}$ in Fig. 3 (circles). Within error, they follow the expected linear relationship. Two additional antibodies were studied, VRC01 and VRC07-523LS, shown as cyan and green triangles in Fig. 3. Within error, they also fall on the same regression line from NISTmAb, suggesting shape-dependent factors to be consistent between these antibodies.

It is possible to examine the hydrodynamic volume in more detail. From the NISTmAb SV data at the lowest protein concentration, which are sufficiently dilute for the ideal sedimentation model to apply, frictional ratios of $\approx 1.6$ were obtained for all conditions. Together with a protein partial-specific volume of $0.73 \mathrm{ml} / \mathrm{g}$, this allows us to calculate the expected $k_{S}$ value for conditions of vanishing virial coefficients (theta condition) considering only the protein partial-specific volume (Eq. 4), termed $k_{0, V}$, or the analogous value considering the volume of the hydrodynamic equivalent sphere, termed $k_{O, H}$. The values for $k_{O, V}$ and $k_{O, H}$ are labeled on the ordinate axis of Fig. 3. From interpolation of the

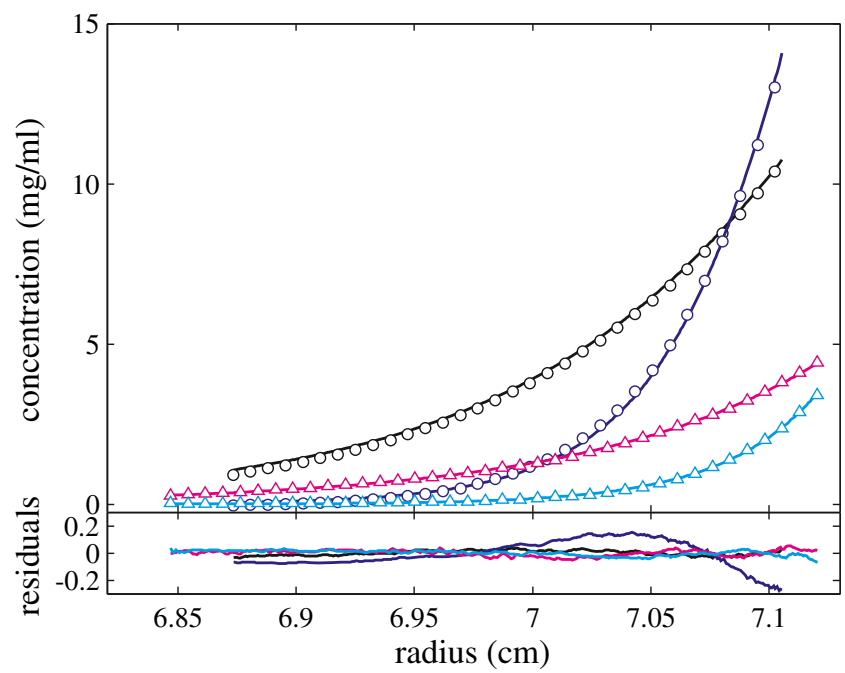

Fig. 1. SE data of NISTmAb in $25 \mathrm{mM}$ histidine pH 6.0 with $5 \mathrm{mM}$ $\mathrm{NaCl}$. Shown are Rayleigh interferometric fringe shift data in a $3 \mathrm{~mm}$ pathlength cell at $9000 \mathrm{rpm}$ and 14,000 rpm (black and blue circles, respectively) and at much lower loading concentration in a $12 \mathrm{~mm}$ pathlength cell at the same rotor speeds (magenta and cyan triangles, values tenfold magnified). Only every 10th data point is shown for clarity. The solid lines are the respective best-fit distribution based on a global analysis of data from 4 different cells. The best-fit buoyant molar mass is $39.2 \mathrm{kDa}$ (corresponding to $\mathrm{a} \approx 150 \mathrm{kDa}$ protein) and the best-fit virial coefficient is $11.6(10.7-12.4) \mathrm{ml} / \mathrm{g}$. The lower panel shows the residuals from the fit with an rmsd of 0.029 fringes 


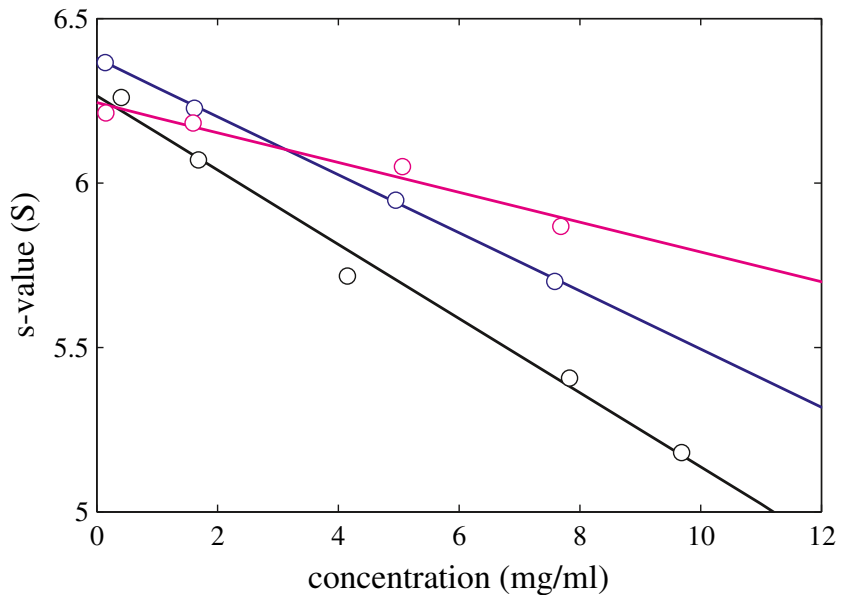

Fig. 2. Experimental sedimentation coefficients of NISTmAb monomer at different conditions. All data are in $25 \mathrm{mM}$ histidine $\mathrm{pH} 6.0$ with 0 (black), $5 \mathrm{mM}$ (blue), or $50 \mathrm{mM} \mathrm{NaCl}$ (magenta). The effective concentrations were calculated from the loading concentrations corrected for slight dilution during the SV run. The line represents the best-fit linear regression. Deviations from the expected straight line fit were used to estimate errors in $s_{w}$ which were propagated to uncertainties in $k_{S}(50)$

experimental NISTmAb data points to the theta conditions

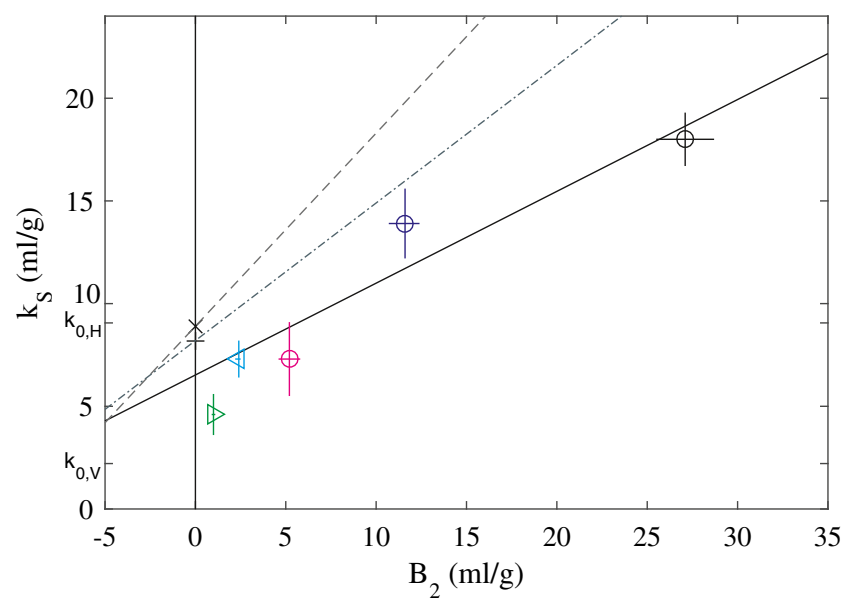

Fig. 3. Relationship between measured $k_{S}$ and $B_{2}$ for the NISTmAb and VRC antibodies studied. Shown are the experimental data points for NISTmAb in formulation condition (black circle) and supplemented with $5 \mathrm{mM}$ (blue circle) or $50 \mathrm{mM} \mathrm{NaCl}$ (magenta circle). The solid line is the linear regression of NISTmAb data $(\mathrm{R}=0.93)$. Additionally shown are data points for VRC01 (cyan triangle) and VRC07-523LS (green triangle). The vertical line indicates the condition where the virial coefficient vanishes and does not make any contribution to the hydrodynamic nonideality of sedimentation (theta condition). The intercept of this line with the interpolated regression may be compared with the theoretical predictions $k_{0, V}(\mathrm{Eq}$. 4) and $k_{0, H}$ (Eq. 6) of accounting for the occupied volume fraction on the basis of the protein partial-specific volume only, or considering the hydrodynamically equivalent spheres, respectively. The $k_{S}$ values calculated based on the regression of the $k_{D}$ vs. $B_{2}$ data of different antibodies measured by DLS/SLS in Lehermayr et al. (30) is shown as short dashed line, and the ones based on a similar regression of different antibodies measured by DLS/SV in Connolly et al. (9) are shown as short dotted line, with the respective values at the theta condition highlighted as cross and plus sign, respectively
$B_{2}=0$ we can derive an experimental estimate of the nonideality of sedimentation for vanishing virial coefficients, $k_{S}^{0}$ (Eq. 7), which exclusively reports on the shape dependence of the hydrodynamic interactions. As may be discerned from Fig. 3, the experimental value is $6.5 \mathrm{ml} / \mathrm{g}$, i.e., in between $k_{0, V}(2.2 \mathrm{ml} / \mathrm{g})$ and $k_{0, H}(9.1 \mathrm{ml} / \mathrm{g})$. The experimental value represents a $\approx 3$-fold volume expansion relative to the merely displaced volume, but only $72 \%$ the volume of the hydrodynamically equivalent spheres.

Interestingly, at this theta point $k_{D}=-k_{S}$, i.e., the nonideality coefficient of diffusion and sedimentation must be of the same magnitude and opposite sign. Therefore, the linear regression of the relationship between $k_{D}$ and second virial coefficient among different antibodies reported by Connolly (9) and those by Lehermayr (30) may be compared at the theta condition, leading to $k_{S}^{0}$ values of $8.2 \mathrm{ml} / \mathrm{g}$ and $8.9 \mathrm{ml} / \mathrm{g}$, respectively (indicated as cross and plus sign in Fig. 3). Similar $k_{S}^{0}$ values at theta conditions of $8-10 \mathrm{ml} / \mathrm{g}$ can be estimated from the study of different antibodies at different solution conditions by Saito and colleagues (15). These values are all close to the value of $9.1 \mathrm{ml} / \mathrm{g}$ predicted for the hydrodynamically equivalent sphere of antibodies. It is possible to further transform the regressions $k_{D}$ vs. $B_{2}$ reported by Connolly (9) into $k_{S}$ vs. $B_{2}$ noting that $k_{S}=$ $2 B_{2}-k_{D}$. As can be discerned from the dotted and short dotted lines in Fig. 3, such transforms are consistent with the present data neither for those measured by Connolly et al. using DLS/SV (9) nor with those measured by Lehermayr based on DLS/SLS (30).

Next, we tested these relationships with a smaller and more compact protein, using hen egg lysozyme as another well-studied model protein. The virial coefficients $B_{2}$ were measured again by SE in different solution conditions, all at $\mathrm{pH} 4.6$ in $10 \mathrm{mM}$ acetate, with different degree of charge screening by $10 \mathrm{mM} \mathrm{NaCl}, 100 \mathrm{mM} \mathrm{NaCl}$, and $300 \mathrm{mM} \mathrm{NaCl}$. Many groups have previously reported decreasing $B_{2}$ with increasing salt (71-73) and our experimental $B_{2}$ values compare favorably with the range of published values $(10,13,72)$.

The SV measurements to determine $k_{S}$ are shown in Fig. 4. The plot $k_{S}$ vs. $B_{2}$ (Fig. 5) shows the expected strong correlation. Due to the more compact shape (best-fit $f / f_{0}=$ 1.18) the $k_{0, V}$ and $k_{0, H}$ are closer together $(2.2 \mathrm{ml} / \mathrm{g}$ and $3.6 \mathrm{ml} / \mathrm{g}$, respectively), with the interpolated experimental value of $k_{S}^{0} 2.9 \mathrm{ml} / \mathrm{g}$ in between the two values.

Lysozyme is known to self-associate dependent on solution $\mathrm{pH}$ and ionic strength, a feature also exhibited by some therapeutic antibodies and other therapeutic proteins $(74,75)$. Self-association is a reflection of attractive proteinprotein interaction and as such well described in the framework of virial coefficients (76-78), which become smaller and even negative, as is the case for lysozyme at pH 4.6 in $300 \mathrm{mM} \mathrm{NaCl}$. Similarly, $k_{\mathrm{S}}$ values decrease as a result of self-association (79). In the case of moderate and strong self-association, due to poor convergence of virial expansion (76), the concentration-dependence of equilibrium properties is better described by explicitly accounting for different oligomeric states in chemical equilibrium. Analogously, it is possible to switch the hydrodynamic analysis to a picture that allows to describe polydispersity from self- 


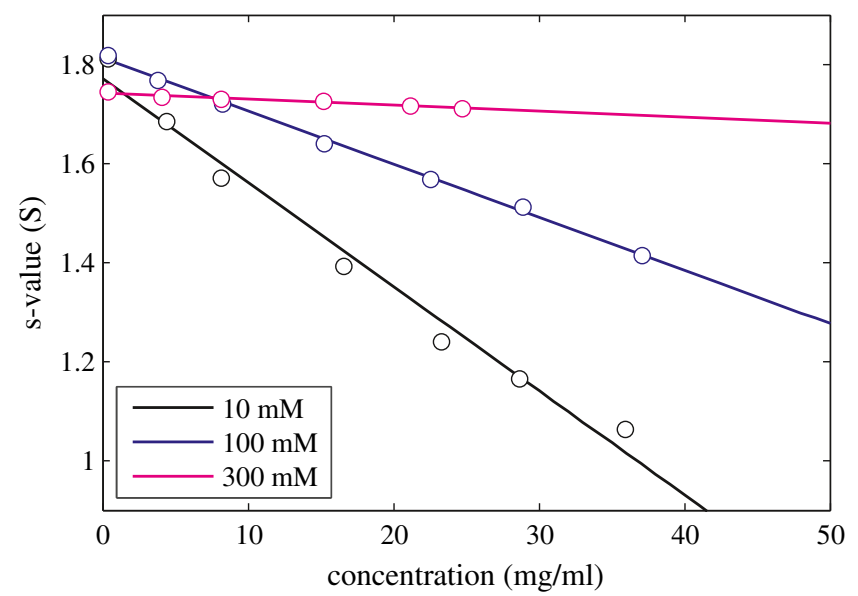

Fig. 4. Experimental sedimentation coefficients of lysozyme at different conditions. All data are at pH 4.6 in $10 \mathrm{mM}$ acetate with different concentration of $\mathrm{NaCl}: 10 \mathrm{mM} \mathrm{NaCl}$ (black), $100 \mathrm{mM} \mathrm{NaCl}$ (blue), and $300 \mathrm{mM} \mathrm{NaCl}$ (magenta). The line represents the best-fit linear regression based on Eq. 1

association separately (42). In this alternative picture, $k_{\mathrm{S}}$ is determined, not from the concentration-dependence of the weight-average $s$ value, but from the nonideal sedimentation coefficient distribution $c_{\mathrm{NI}}\left(s_{0}\right)$. These resulting $k_{S}$ data (shown in green in Fig. 5, taken from (42)) are found also to exhibit a linear relationship with $B_{2}$.

Finally, a single experiment with ovalbumin in PBS is shown in Fig. 5 as cyan triangle. This protein has a different structure and a different shape factor (with a best-fit frictional

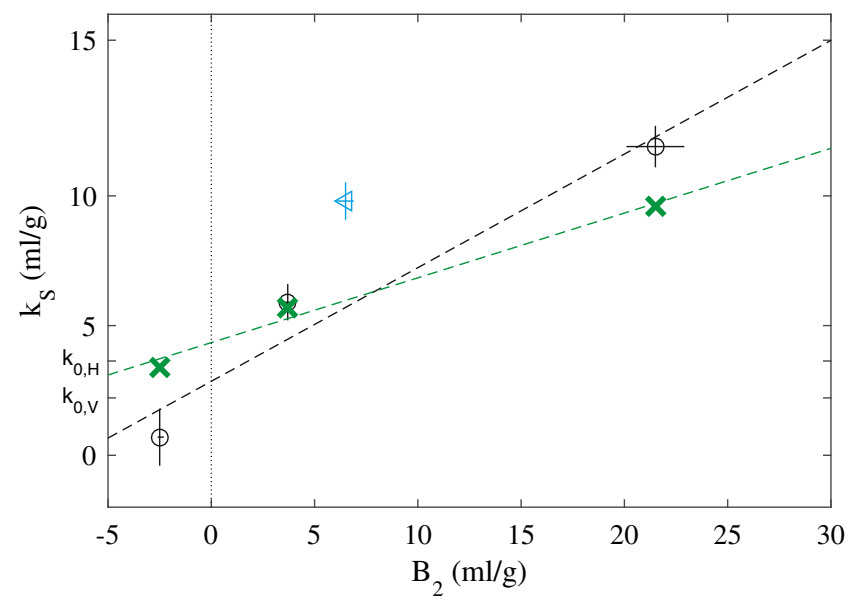

Fig. 5. Relationship between measured $k_{S}$ and $B_{2}$ for hen egg lysozyme. Shown are the experimental data points for lysozyme in $10 \mathrm{mM}$ sodium acetate $\mathrm{pH} 4.6$ with $10 \mathrm{mM}, 100 \mathrm{mM}$, or $300 \mathrm{mM} \mathrm{NaCl}$ (circles). The dashed black line is the linear regression $(R=0.97)$. Indicated on the ordinate are the theoretical predictions $k_{0, V}$ and $k_{0, H}$ at the theta condition when accounting for the occupied volume fraction on the basis of the protein partial-specific volume only, or considering the hydrodynamically equivalent spheres, respectively. The green data points reflect $k_{S}$ values derived from a sedimentation coefficient distribution $c_{\mathrm{NI}}\left(s_{0}\right)$ that accounts separately for polydispersity from lysozyme self-association (taken from (42) for the $100 \mathrm{mM}$ and $300 \mathrm{mM} \mathrm{NaCl}$ conditions), with linear regression $(R=$ 0.992; dashed green line). Additionally shown are data from ovalbumin in PBS (cyan triangle) ratio of 1.24) and it does not fall on the same line as either lysozyme or NISTmAb. Similarly, neither the experimental $k_{S^{-}} B_{2}$ relationships of lysozyme nor NISTmAb are consistent with each other. This supports the notion that the precise relationship will be dependent on the specific protein shape.

\section{DISCUSSION}

The choice of techniques for measuring the second virial coefficient depends on many factors, including purity, experimental time, concentration range, and volumes. In the present work, we have focused on AUC approaches. Among all techniques for measuring the second virial coefficient, traditional analytical ultracentrifugation may be one of the lowest throughput methods. However, a significant advantage is the intrinsic size separation: large particles will quickly sediment and not impact the measurement. This makes it a very attractive approach for samples that might potentially contain traces of higher-order structure oligomers or particles.

As Saito and co-workers (15) have pointed out, SE is attractive due to its relatively small sample volumes and the ability to study higher concentrations than the loading concentration due to the accumulation of protein in the distal end of the solution column afforded by the gravitational field. In order to facilitate SE experiments, we have previously introduced a timevarying centrifugal field approach that can shorten the time required to reach equilibrium (48). In the present, work we have implemented a new sedimentation model that allows determination of $B_{2}$ across a wider concentration range. It accounts for higher-order virial coefficients through relationships based on effective hard spheres, and due to the higher concentration, the range should provide higher precision in $B_{2}$.

The main focus of the present communication is to highlight an opportunity to measure virial coefficients directly by SV exploiting a relationship from statistical fluid mechanics that predicts the magnitude of $k_{S}$ in the presence of weak particle interactions. Within error, our data confirm the linear relationship between $k_{S}$ and $B_{2}$. They also highlight the difficulty of quantitatively interpreting the absolute magnitude of $k_{S}$, due to uncertainties in the relevant hydrodynamic volume, although our data suggest the volume of the Stokes (hydrodynamic equivalent) sphere may be the best estimate. More precise studies in this area would be plagued by the impossibility to vary solution conditions without simultaneously varying the effective sedimenting particle including hydration, sedimenting counterions, and conformation (80), and may be limited by simplified models of particle shapes $(80,81)$. The interpretation of the absolute value of $k_{S}$, even for spherical particles such as polystyrene latex beads (59), is non-trivial due to the unknown contributions of weak interactions (82).

Pragmatically, however, questions regarding the absolute values of $k_{S}$ should not impact the application of SV to screen and compare different buffer conditions. One could argue that absolute values for $B_{2}$ may be hard to dissect into steric contributions and those from weak interactions and that relative values $B_{2} / B_{H S}$ may sufficiently reflect on the differences of weak interactions in different buffer conditions. Finally, it appears that if $k_{D}$ may be used to assess differences in $B_{2}$ and exhibits a linear relationship, as proposed by Connolly and colleagues (9), then $k_{S}$ must likewise exhibit a linear relationship with $B_{2}$ (since $k_{S}=$ $2 B_{2}-k_{D}$ ). Our data support the conclusion by Connolly and 
colleagues that $k_{S}$ values should be compared only for the same molecule. Which technique provides better approach may depend on several factors including relative accuracy and sample properties. Two very recent developments have significantly enhanced SV. One is the ability to measure in a single experiment at high concentration (e.g., up to $80 \mathrm{mg} / \mathrm{ml}$ of $\gamma$ crystallin) of unlabeled material the nonideality coefficient alongside the sedimentation coefficient distribution (29). This is synergistic with the application of SV to quantify higher-order structures, such as oligomers and aggregates, in different conditions (29,38-41). Second, we have developed threechannel centerpieces that allow doubling the capacity of SV samples in an AUC run and using smaller sample volumes (manuscript submitted). This can greatly improve the throughput of SV, extending the number of conditions that can be examined in a single run from 1 or 2 to up to 15.

As pointed out by Roberts and co-workers (28), there are inherent limitations of measuring protein properties in a relatively low concentration regime to predict behavior at much higher concentration. In future work, making use of recent developments in theory describing sedimentation at higher concentrations beyond the first-order nonideality approximation (36), detection technology (83-85), and centerpiece design (44) (manuscript in preparation), it may be possible to extend SV experiments further to highly concentrated formulation conditions measuring both weak interactions and sample polydispersity.

\section{ACKNOWLEDGEMENTS}

We thank Dr. Huaying Zhao for helpful discussions, and we are grateful to Drs. Jai Pathak, Lisa Kueltzo, and Frank Arnold for providing the VRC antibodies.

\section{FUNDING INFORMATION}

This work was supported by the Intramural Research Program of the National Institute of Biomedical Imaging and Bioengineering, National Institutes of Health.

Open Access This article is distributed under the terms of the Creative Commons Attribution 4.0 International License (http://creativecommons.org/licenses/by/4.0/), which permits unrestricted use, distribution, and reproduction in any medium, provided you give appropriate credit to the original author(s) and the source, provide a link to the Creative Commons license, and indicate if changes were made.

Publisher's Note Springer Nature remains neutral with regard to jurisdictional claims in published maps and institutional affiliations.

\section{REFERENCES}

1. Beck A, Wurch T, Bailly C, Corvaia N. Strategies and challenges for the next generation of therapeutic antibodies. Nat Rev Immunol. 2010;10(5):345-52.

2. Brinkmann U, Kontermann RE. The making of bispecific antibodies. MAbs. 2017;9(2):182-212.
3. Walsh G. Biopharmaceutical benchmarks 2014. Nat Biotechnol. 2014;32(10):992-1000.

4. Roberts CJ. Protein aggregation and its impact on product quality. Curr Opin Biotechnol. 2014;30:211-7.

5. Uchiyama S. Liquid formulation for antibody drugs. Biochim Biophys Acta - proteins. Proteomics. 2014;1844(11):2041-52.

6. Shire SJ, Shahrokh Z, Liu J. Challenges in the development of high protein concentration formulations. J Pharm Sci. 2004;93:1390-402.

7. Zbacnik TJ, Holcomb RE, Katayama DS, Murphy BM, Payne RW, Coccaro RC, et al. Role of buffers in protein formulations. J Pharm Sci. 2017;106(3):713-33.

8. Zhao H, Brown $\mathrm{PH}$, Balbo A, Fernandez Alonso MC, Polishchuck N, Chaudhry C, et al. Accounting for solvent signal offsets in the analysis of interferometric sedimentation velocity data. Macromol Biosci. 2010;10(7):736-45.

9. Connolly BD, Petry C, Yadav S, Demeule B, Ciaccio N, Moore JMR, et al. Weak interactions govern the viscosity of concentrated antibody solutions: high-throughput analysis using the diffusion interaction parameter. Biophys J. 2012;103(1):69-78.

10. Saluja A, Fesinmeyer RM, Hogan S, Brems DN, Gokarn YR. Diffusion and sedimentation interaction parameters for measuring the second virial coefficient and their utility as predictors of protein aggregation. Biophys J. 2010;99(8):2657-65.

11. Raut AS, Kalonia DS. Pharmaceutical perspective on opalescence and liquid-liquid phase separation in protein solutions. Mol Pharm. 2016;13(5):1431-44.

12. Rosenbaum DF, Zukoski CF. Protein interactions and crystallization. J Cryst Growth. 1996;169(4):752-8.

13. Quigley A, Williams DR. The second virial coefficient as a predictor of protein aggregation propensity: a self-interaction chromatography study. Eur J Pharm Biopharm. 2015;96:282-90.

14. Baek Y, Zydney AL. Intermolecular interactions in highly concentrated formulations of recombinant therapeutic proteins. Curr Opin Biotechnol. 2018;53:59-64.

15. Saito S, Hasegawa J, Kobayashi N, Kishi N, Uchiyama S, Fukui $\mathrm{K}$. Behavior of monoclonal antibodies: relation between the second virial coefficient (B 2) at low concentrations and aggregation propensity and viscosity at high concentrations. Pharm Res. 2012;29(2):397-410.

16. Solovyova A, Schuck P, Costenaro L, Ebel C. Non-ideality by sedimentation velocity of halophilic malate dehydrogenase in complex solvents. Biophys J. 2001;81(4):1868-80.

17. Rivas G, Minton AP. Beyond the second virial coefficient: sedimentation equilibrium in highly non-ideal solutions. Methods. 2011;54(1):167-74.

18. Minton AP. Static light scattering from concentrated protein solutions, I: general theory for protein mixtures and application to self-associating proteins. Biophys J. 2007;93(4):1321-8.

19. Moon YU, Curtis RA, Anderson CO, Blanch HW, Prausnitz JM. Protein-protein interactions in aqueous ammonium sulfate solutions. Lysozyme and bovine serum albumin (BSA). J Solut Chem. 2000;29(8):699-717.

20. Yearley EJ, Godfrin PD, Perevozchikova T, Zhang H, Falus P, Porcar L, et al. Observation of small cluster formation in concentrated monoclonal antibody solutions and its implications to solution viscosity. Biophys J. 2014;106(8):1763-70.

21. Ang S, Rowe AJ. Evaluation of the information content of sedimentation equilibrium data in self-interacting systems. Macromol Biosci. 2010;10(7):798-807.

22. Castellanos MM, Mattison K, Krueger S, Curtis JE. Characterization of the NISTmAb reference material using small-angle scattering and molecular simulation. Anal Bioanal Chem. 2018;410(8):2161-71.

23. Tessier PM, Lenhoff AM, Sandler SI. Rapid measurement of protein osmotic second virial coefficients by self-interaction chromatography. Biophys J. 2002;82(3):1620-31.

24. Blanco MA, Sahin E, Li Y, Roberts CJ. Reexamining proteinprotein and protein-solvent interactions from Kirkwood-buff analysis of light scattering in multi-component solutions. J Chem Phys. 2011;134(22):225103.

25. Ma Y, Acosta DM, Whitney JR, Podgornik R, Steinmetz NF, French RH, et al. Determination of the second virial coefficient of bovine serum albumin under varying $\mathrm{pH}$ and ionic strength by composition-gradient multi-angle static light scattering. J Biol Phys. 2015;41(1):85-97. 
26. Courtenay ES, Capp MW, Anderson CF, Record MT. Vapor pressure osmometry studies of osmolyte-protein interactions: implications for the action of osmoprotectants in vivo and for the interpretation of "osmotic stress" experiments in vitro. Biochemistry. 2000;39(15):4455-71.

27. Ghosh R, Calero-Rubio C, Saluja A, Roberts CJ. Relating protein-protein interactions and aggregation rates from low to high concentrations. J Pharm Sci. 2016;105(3):1086-96.

28. Calero-Rubio C, Ghosh R, Saluja A, Roberts CJ. Predicting protein-protein interactions of concentrated antibody solutions using dilute solution data and coarse-grained molecular models. J Pharm Sci. 2018;107(5):1269-81.

29. Chaturvedi SK, Ma J, Brown PH, Zhao H, Schuck P. Measuring macromolecular size distributions and interactions at high concentrations by sedimentation velocity. Nat Commun. 2018;9(1):4415

30. Lehermayr C, Mahler H-C, Mäder K, Fischer S. Assessment of net charge and protein-protein interactions of different monoclonal antibodies. J Pharm Sci. 2011;100(7):2551-62.

31. Harding SE, Johnson P. The concentration-dependence of macromolecular parameters. Biochem J. 1985;231(3):543-7.

32. Schuck P, Zhao H. Sedimentation velocity analytical ultracentrifugation: interacting systems. Boca Raton: CRC Press; 2017. $271 \mathrm{p}$

33. Lattuada E, Buzzaccaro S, Piazza R. Colloidal swarms can settle faster than isolated particles: enhanced sedimentation near phase separation. Phys Rev Lett. 2016;116(3):038301.

34. Russel WB, Saville DA, Schowalter WR. Colloidal Dispersions. Cambridge: Cambridge University Press; 1989.

35. Moncho-Jordá A, Louis AA, Padding JT. Effects of interparticle attractions on colloidal sedimentation. Phys Rev Lett. 2010;104(6):068301.

36. Fiore AM, Wang G, Swan JW. From hindered to promoted settling in dispersions of attractive colloids: simulation, modeling, and application to macromolecular characterization. Phys Rev Fluids. 2018;3(6):063302.

37. Batchelor GK, Wen C-S. Sedimentation in a dilute polydisperse system of interacting spheres. Part 2. Numerical results. J Fluid Mech. 1982;124:495-528.

38. Gandhi AV, Pothecary MR, Bain DL, Carpenter JF. Some lessons learned from a comparison between sedimentation velocity analytical ultracentrifugation and size exclusion chromatography to characterize and quantify protein aggregates. J Pharm Sci. 2017;106(8):2178-86.

39. Berkowitz SA. Role of analytical ultracentrifugation in assessing the aggregation of protein biopharmaceuticals. AAPS J. 2006;8(3):E590-605.

40. Liu J, Yadav S, Andya J, Demeule B, Shire SJ. Analytical ultracentrifugation and its role in development and research of therapeutical proteins. Methods Enzymol. 2015;562:44176.

41. Arthur KK, Gabrielson JP, Kendrick BS, Stoner MR. Detection of protein aggregates by sedimentation velocity analytical ultracentrifugation (SV-AUC): sources of variability and their relative importance. J Pharm Sci. 2009;98(10):3522-39.

42. Chaturvedi SK, Sagar V, Zhao H, Wistow G, Schuck P. Measuring ultra-weak protein self-association by nonideal sedimentation velocity. J Am Chem Soc. 2019:jacs.8b11371.

43. Kar SR, Kingsbury JS, Lewis MS, Laue TM, Schuck P. Analysis of transport experiments using pseudo-absorbance data. Anal Biochem. 2000;285(1):135-42.

44. Desai A, Krynitsky J, Pohida TJ, Zhao H, Schuck P. 3D-printing for analytical ultracentrifugation. PLoS One. 2016;11(8): 0155201.

45. Schiel JE, Turner A, Mouchahoir T, Yandrofski K, Telikepalli S, King J, et al. The NISTmAb reference material 8671 value assignment, homogeneity, and stability. Anal Bioanal Chem. 2018;410(8):2127-39.

46. Schuck P, Zhao H, Brautigam CA, Ghirlando R. Basic principles of analytical ultracentrifugation. Boca Raton: CRC Press; 2015. $302 \mathrm{p}$

47. Zhao H, Brautigam CA, Ghirlando R, Schuck P. Overview of current methods in sedimentation velocity and sedimentation equilibrium analytical ultracentrifugation. Curr Protoc Protein Sci. 2013;7(71):20.12.1.
48. Ma J, Metrick M, Ghirlando R, Zhao H, Schuck P. Variablefield analytical ultracentrifugation: I. Time-optimized sedimentation equilibrium. Biophys J. 2015;109(4):827-37.

49. Johnson ML. Why, when, and how biochemists should use least squares. Anal Biochem. 1992;225:215-25.

50. Bevington PR, Robinson DK. Data reduction and error analysis for the physical sciences. New York: Mc-Graw-Hill; 1992.

51. Schuck P. Size-distribution analysis of macromolecules by sedimentation velocity ultracentrifugation and Lamm equation modeling. Biophys J. 2000;78(3):1606-19.

52. Schuck P. Sedimentation velocity analytical ultracentrifugation: discrete species and size-distributions of macromolecules and particles. Boca Raton: CRC Press; 2016. 244 p

53. Schuck P. On the analysis of protein self-association by sedimentation velocity analytical ultracentrifugation. Anal Biochem. 2003;320(1):104-24.

54. Patel TR, Winzor DJ, Scott DJ. Allowance for radial dilution in evaluating the concentration dependence of sedimentation coefficients for globular proteins. Eur Biophys J. 2017;47:291-5.

55. Ghirlando R, Balbo A, Piszczek G, Brown PH, Lewis MS, Brautigam CA, et al. Improving the thermal, radial, and temporal accuracy of the analytical ultracentrifuge through external references. Anal Biochem. 2013;440(1):81-95.

56. Zhao H, Ghirlando R, Alfonso C, Arisaka F, Attali I, Bain DL, et al. A multilaboratory comparison of calibration accuracy and the performance of external references in analytical ultracentrifugation. PLoS One. 2015;10(5):e0126420.

57. Batchelor GK. Sedimentation in a dilute dispersion of spheres. J Fluid Mech. 1972;52(2):245-68.

58. Batchelor GK. Sedimentation in a dilute polydisperse system of interacting spheres. 1. General theory. J Fluid Mech. 1982;119:379-408.

59. Cheng PY, Schachman HK. Studies on the validity of the Einstein viscosity law and stokes law of sedimentation. J Polym Sci. 1955;16(81):19-30.

60. Dogic Z, Philipse AP, Fraden S, Dhont JKG. Concentrationdependent sedimentation of colloidal rods. J Chem Phys. 2000;113(18):8368-80.

61. Claeys IL, Brady JF. Suspensions of prolate spheroids in stokes flow. Part 1 . Dynamics of a finite number of particles in an unbounded fluid. J Fluid Mech. 1993;251:411-42.

62. Creeth JM, Knight CG. On the estimation of the shape of macromolecules from sedimentation and viscosity measurements. Biochim Biophys Acta. 1965;102(2):549-58.

63. Pavlov GM, Rowe AJ, Harding SE. Conformation zoning of large molecules using the analytical ultracentrifuge zones. Trends Anal Chem. 1997;16(7):401-5.

64. Rowe AJ. The concentration dependence of transport processes: a general description applicable to the sedimentation, translational diffusion, and viscosity coefficients of macromolecular solutes. Biopolymers. 1977:16:2595-611.

65. Calero-Rubio C, Saluja A, Roberts CJ. Coarse-grained antibody models for "weak" protein-protein interactions from low to high concentrations. J Phys Chem B. 2016;120(27):6592-605.

66. Chatelier RC, Minton AP. Sedimentation equilibrium in macromolecular solutions of arbitrary concentration. I. Selfassociating proteins. Biopolymers. 1987;26(4):507-24.

67. Carnahan NF, Starling KE. Equation of state for nonattracting rigid spheres. J Chem Phys. 1969;51(2):635-6.

68. Minton AP. Molecular crowding: analysis of effects of high concentrations of inert cosolutes on biochemical equilibria and rates in terms of volume exclusion. Methods Enzymol. 1998;295:127-49.

69. Vistica J, Dam J, Balbo A, Yikilmaz E, Mariuzza RA, Rouault TA, et al. Sedimentation equilibrium analysis of protein interactions with global implicit mass conservation constraints and systematic noise decomposition. Anal Biochem. 2004;326(2):234-56.

70. Gohon Y, Agarwal S, Arthur K, Bepperling A, Day ES, Filoti $\mathrm{D}$, et al. Biophysical techniques for characterizing the higher order structure and interactions of monoclonal antibodies. In: Schiel J, Davis D, Borisov O, editors. State-of-the-art and emerging Technologies for Therapeutic Monoclonal Antibody Characterization Volume 2 biopharmaceutical characterization: the NISTmAb case study: American Chemical Society; 2015. p. 285-327. 
71. Muschol M, Rosenberger F. Interactions in undersaturated and supersaturated lysozyme solutions: static and dynamic light scattering results. J Chem Phys. 1995;103(24):10424-32.

72. Velev OD, Kaler EW, Lenhoff AM. Protein interactions in solution characterized by light and neutron scattering: comparison of lysozyme and Chymotrypsinogen. Biophys J. 1998;75(6):2682-97.

73. Valente JJ, Verma KS, Manning MC, Wilson WW, Henry CS. Second virial coefficient studies of cosolvent-induced protein self-interaction. Biophys J. 2005;89(6):4211-8.

74. Hopkins MM, Lambert CM, Bee JS, Parupudi A, Bain DL. Determination of interaction parameters for reversibly selfassociating antibodies: a comparative analysis. J Pharm Sci. 2018;107(7):1820-30.

75. Sule SV, Sukumar M, Weiss WF IV, Marcelino-Cruz AM, Sample T, Tessier PM. High-throughput analysis of concentration-dependent antibody self-association. Biophys J. 2011;101(7):1749-57.

76. Stafford WF, Yphantis DA. Virial expansions for ideal selfassociating systems. Biophys J. 1972;12(10):1359-65.

77. Wills PR. A hilly path through the thermodynamics and statistical mechanics of protein solutions. Biophys Rev. 2016;8(4):291-8.

78. Hill TL, Chen Y-D. Theory of aggregation in solution. I. General equations and application to the stacking of bases, nucleosides, etc. Biopolymers. 1973;12(6):1285-312.
79. Yang D, Correia JJ, Stafford WF, Roberts CJ, Singh S, Hayes D, et al. Weak IgG self- and hetero-association characterized by fluorescence analytical ultracentrifugation. Protein Sci. 2018;27(7):1334-48.

80. Myung JS, Roosen-Runge F, Winkler RG, Gompper G, Schurtenberger P, Stradner A. Weak shape anisotropy leads to a non-monotonic contribution to crowding impacting protein dynamics under physiologically relevant conditions. J Phys Chem B. 2018:acs.jpcb.8b07901.

81. Claeys IL, Brady JF. Suspensions of prolate spheroids in stokes flow. Part 2 . Statistically homogeneous dispersions. J Fluid Mech. 1993;251:443-77.

82. Goldstein B, Zimm BH. Effect of concentration and intermolecular forces on the sedimentation of polystyrene spheres. J Chem Phys. 1971;54(10):4408-13.

83. Schilling K, Krause F. Analysis of antibody aggregate content at extremely high concentrations using sedimentation velocity with a novel interference optics. PLoS One. 2015;10(3):e0120820.

84. Wawra SE, Pflug L, Thajudeen T, Kryschi C, Stingl M, Peukert W. Determination of the two-dimensional distributions of gold nanorods by multiwavelength analytical ultracentrifugation. Nat Commun. 2018;(Icmm):1-11.

85. Kroe RR, Laue TM. NUTS and BOLTS: applications of fluorescence-detected sedimentation. Anal Biochem. 2009;390(1):1-13. 